Journal of Quantitative Spectroscopy \& Radiative Transfer 91 (2005) 65-93
Journal of Quantitative Spectroscopy \& Radiative Transfer

www.elsevier.com/locate/jqsrt

\title{
ARTS, the atmospheric radiative transfer simulator
}

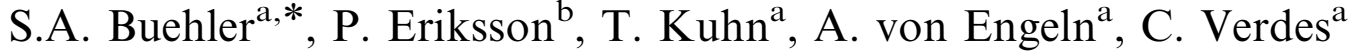 \\ ${ }^{a}$ Institute of Environmental Physics, University of Bremen, Otto-Hahn-Allee 1, D-28359 Bremen, Germany \\ ${ }^{\mathrm{b}}$ Department of Radio and Space Science, Chalmers University of Technology, SE-41296 Gothenburg, Sweden
}

Received 30 July 2003; accepted 21 May 2004

\begin{abstract}
ARTS is a modular program that simulates atmospheric radiative transfer. The paper describes ARTS version 1.0, which is applicable in the absence of scattering. An overview over all major parts of the model is given: calculation of absorption coefficients, the radiative transfer itself, and the calculation of Jacobians. ARTS can be freely used under a GNU general public license.

Unique features of the program are its scalability and modularity, the ability to work with different sources of spectroscopic parameters, the availability of several self-consistent water continuum and line absorption models, and the analytical calculation of Jacobians.

(C) 2004 Elsevier Ltd. All rights reserved.
\end{abstract}

Keywords: Radiative transfer; Radiative transfer Jacobians; Gaseous absorption; Spectroscopic databases

\section{Introduction}

The number of satellite sensors in the millimetre and sub-millimetre spectral range is rapidly growing. They use various frequency bands and observation geometries. Two important groups of sensors are for example the down looking millimetre wave sensors like the Advanced Microwave Sounding Unit (AMSU), and the limb looking sub-millimetre wave sensors like the planned Superconducting Sub-Millimetre Wave Limb Emission Sounder (SMILES).

\footnotetext{
${ }^{*}$ Corresponding author. Tel.: +46-31-772-4417; fax: +46-31-772-4555.

E-mail address: sbuehler@uni-bremen.de (S.A. Buehler).
} 
For the data analysis, all such sensors require accurate and fast forward models, which can simulate the measurement corresponding to a given atmospheric state. Depending on the objective of the sensor, the measurement will depend for example on the distribution of atmospheric temperature, water vapour, ozone, and many other trace gases. It may also depend on surface properties, particularly for down looking sensors in atmospheric 'windows'.

This paper describes the atmospheric radiative transfer simulator ARTS, a tool fast enough for operational use, but yet flexible enough to allow easy modifications for new sensor characterisation studies. The next section describes the underlying concept. Section 3 describes the calculation of absorption coefficients, Section 4 the radiative transfer, and Section 5 the calculation of Jacobians.

\section{Concept}

\subsection{History}

In the remote sensing scientific community, a lot of effort has been put in developing dedicated forward models for different sensors, although all these models have many features in common. While appropriate for operational data analysis, such specialised models are not appropriate for scientific studies of new sensor concepts, since they cannot easily be adapted to new sensors. This has lead to the development of more general purpose forward models in the work groups of the authors. In Bremen, the main tool was for some years the program FORWARD [1], which was written mostly by Joerg Langen in the time period 1991-1998. In Sweden, the Skuld model [2] was developed during 1997-1998, mainly by Patrick Eriksson. Although these models were rather general and have been used successfully over the years, both suffered from being not easily modifiable and extendable. Hence, it became clear that it would be necessary to develop a new model which emphasises modularity, extendibility, and generality.

It was decided that the development work should be shared between the Bremen and Chalmers universities, with Bremen being largely responsible for the overall program architecture and the absorption part, Chalmers being largely responsible for the radiative transfer part and the calculation of Jacobians. The project was put under a GNU general public license [3], in order to give the right legal framework for such a true collaboration.

The program, along with extensive documentation, is freely available on the Internet, under http://www.sat.uni-bremen.de/arts/. This paper describes the stable 1-0-x branch of the program. Stable means that there will be only bug fixes, no additions of new features. The current ARTS version in this branch at the time of writing is arts-1-0-95. There is also a development branch, 1-1-x, which can handle scattering in the atmosphere. It can be found at the same web address.

There are three important components of documentation for the program: online help, automatic source code documentation, and user guide. The online help can be used to get quick information of variables and functions in the program, which is useful when constructing a control file. Automatic source code documentation is done with the DOXYGEN package [4]. It documents all functions, along with their input and output variables. The result is an HTML 
document that is available online at the ARTS web site. However, the most important component of the documentation is the ARTS User Guide [5]. For the 1-0-x branch it has already more than 200 pages describing in detail the algorithms used, the implementation, and the use of the program.

\subsection{Scope}

The ARTS-1-0-x version discussed in this article is limited to cases where scattering can be neglected and local thermodynamic equilibrium applies. At millimetre and sub-millimetre wavelengths these assumptions are valid from the troposphere up to the mesosphere, but only in the clear-sky case, i.e., in the absence of hydrometeors such as large ice crystals or rain.

The model carries out scalar radiative transfer calculations, that means it treats only the first component of the Stokes vector, corresponding to the total intensity. This is a good approximation in the absence of polarisation effects. The only sources of polarisation effects in the atmosphere are scattering, which has already been excluded, and Zeeman splitting of some spectral lines due to the Earths magnetic field. Hence, the scalar treatment implies that Zeeman effects cannot be modelled explicitly.

The model assumes a one-dimensional spherical atmosphere, in other words, the atmosphere is assumed to be spherically symmetric, with all parameters varying as a function of the vertical coordinate only. The primary vertical coordinate is pressure. All other quantities, such as temperature, geometric altitude, and trace gas concentrations, are given on pressure grids.

ARTS has been developed having passive emission measurements in mind, but pure transmission measurements are also handled. ${ }^{1}$ The model can be used to simulate measurements for any observation geometry: up looking, down looking, or limb looking, and for any sensor position: on the ground, inside the atmosphere, or on a satellite.

The model works with arbitrary frequency grids; hence, it can be used both for the simulation of high-resolution sensors, and for the simulation of broad frequency ranges. The applicable spectral range is from the microwave up to the thermal infrared, but the model is currently only well validated below roughly $1 \mathrm{THz}$. In that frequency range, particular care has been taken to make the absorption calculation consistent with state-of-the-art continuum models for water vapour and nitrogen, and with continuum and line mixing models for oxygen.

Besides providing sets of spectra, ARTS can calculate Jacobians for a number of variables. Analytical expressions are used to calculate Jacobians for trace gas concentrations, continuum absorption, and ground emissivity. Perturbations are used to calculate Jacobians for pointing and frequency offsets, and spectroscopic parameters. For temperature Jacobians, the user can choose between an analytical method, which does not assume hydrostatic equilibrium, and a perturbation method, which does assume hydrostatic equilibrium.

ARTS itself calculates only monochromatic pencil beam radiances, no sensor characteristics are included. The sensor part is covered by a set of Matlab functions that make use of a matrix vector formalism (see Section 2.5).

\footnotetext{
${ }^{1}$ Pure transmission measurements means that the atmospheric emission can be neglected. This is the case for occultation measurements towards the sun or an active source.
} 


\subsection{A modular approach}

The most important notion in ARTS is the workspace. All physical quantities (for example absorption coefficients) are workspace variables (WSVs). WSVs can also be of a more technical nature, for example various grids.

The program performs a calculation by executing a list of workspace methods (WSMs), which are specified in a control file. These WSMs take WSVs as input, and generate WSVs as output. Additional input parameters can be specified as keyword parameters in the control file (Fig. 1).

It is important to note that the control file has a well-defined syntax, which is understood by the ARTS parser. Thus, it is easy to add new WSVs and new WSMs. The program has two internal lookup tables, one for all WSVs and one for all WSMs. The WSM table also lists input WSVs, output WSVs, and keyword parameters for each WSM. To add a new WSM, one just has to add an entry to the lookup table, and write the code for the WSM itself. No further changes to the program are necessary. In particular, no changes to the program logic or to the parser.

The internal lookup tables of WSMs and WSVs have the additional advantage, that command line options for online documentation of each WSV and each WSM were easy to implement. This form of online documentation has proven to be very useful in the daily work with the program.

The chosen approach is modular, because each WSV and each WSM stands by itself, and has its own documentation. It is relatively safe, because formal dependencies are checked before the calculation is performed. For example, a WSM requiring absorption coefficients as input will not run if the WSV holding absorption coefficients is not present.

However, the approach is not 'idiot proof', since the program has no intelligence on the meta level. That is, it is not checked whether the control file makes sense, as long as all the formal dependencies are fulfilled. For example, the program does not stop the user from calculating absorption coefficients twice for the same species, if the appropriate WSMs are put in the control file. This behaviour is considered as a feature, rather than a bug, because the authors' personal experience is that even exotic combinations may make sense in some context. Making them impossible would restrict the power of the program. So, ARTS just assumes that the user knows what he/she is doing.

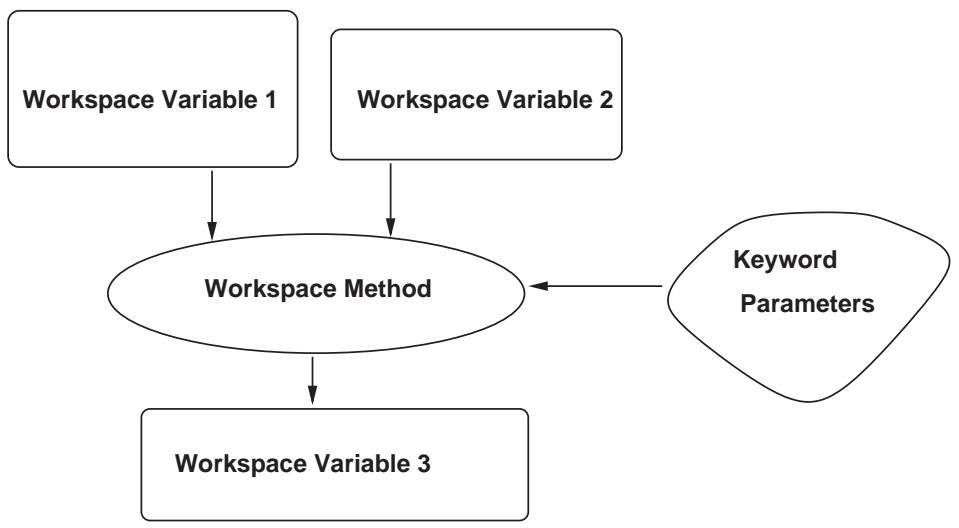

Fig. 1. A workspace method (WSM) acts on some workspace variables (WSVs) to generate other WSVs. Additional input parameters can be specified as keyword parameters in the control file. 


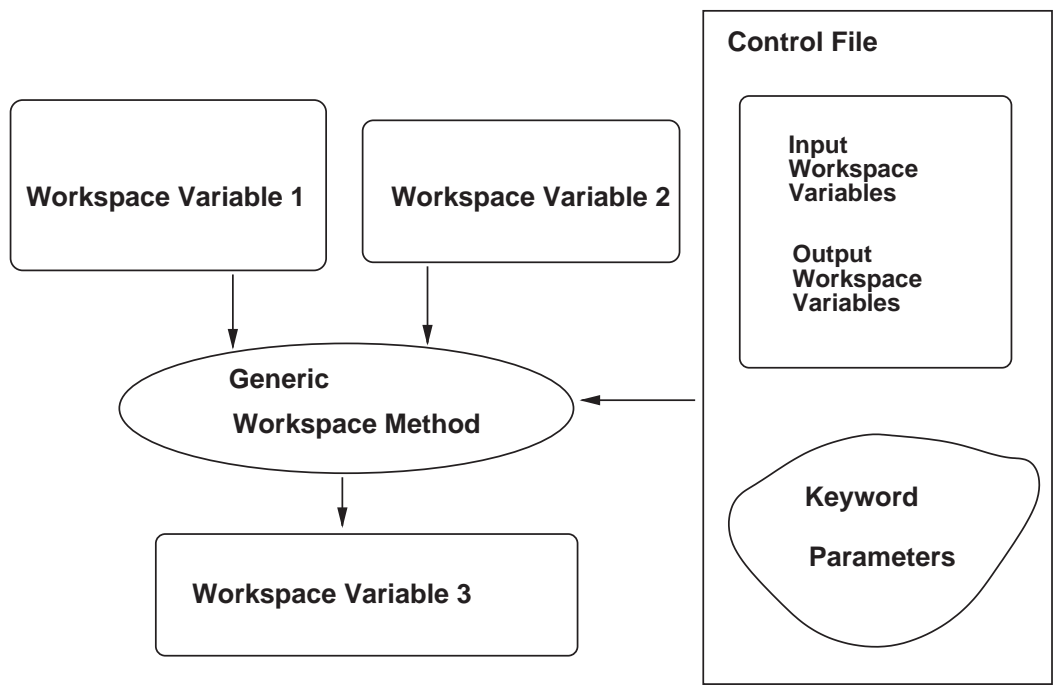

Fig. 2. For generic workspace methods the workspace variables to act on are specified in the control file. The example method discussed in the text takes as keyword parameter in curly braces the name of a file to read, and as input in round braces the name of the output WSV, to which the content of the file should be stored.

\subsection{Generic workspace methods}

Generic WSMs (Fig. 2) allow the user of the program even more freedom than the specific WSMs that were described in the last section. A generic WSM is for example VectorReadAscii, which can be used to read any WSV which is a vector from an ASCII file. For example, the code

VectorReadAscii(f_mono)\{“freqeuency_grid.aa" \}

in the control file will read the specified file and generate the WSV f_grid.

Generic WSMs are particularly useful for IO operations as in the example above. No new IO code is necessary for new WSVs, as long as they are of standard types already known to the program (for example vectors or matrices).

\subsection{Implementation}

The program has been implemented in the $\mathrm{C}++$ programming language, using the standard GNU development tools autoconf and automake. It has been installed and used on different Unix and Linux systems with different versions of the GNU gcc compiler. Table 1 gives a summary of the tested platforms and compiler versions.

For Matlab users there exist two accompanying packages called AMI (ARTS Matlab Interface), and Qpack, ${ }^{2}$ which extend the functionality of ARTS considerably. First of all, AMI has functions to include sensor characteristics in the calculations. AMI has further functions to read and write ARTS data files, and various functions that are of general use. Qpack is a Matlab

\footnotetext{
${ }^{2} \mathrm{AMI}$ is distributed together with ARTS, while Qpack is a separate package.
} 
Table 1

Tested platforms and compiler versions for ARTS-1-0-x

\begin{tabular}{ll}
\hline OS/Architecture & Compiler \\
\hline FreeBSD $4.5 \times 86$ & gcc 2.95 .3 \\
HP Unix 11 & gcc 3.0 .2 \\
Mandrake Linux $9.1 \times 86$ & gcc 3.2 \\
RedHat Linux $7.3 \times 86$ & gcc 2.96 \\
SuSE Linux $7.0 \times 86$ & gcc $2.95 .2 / 2.95 .3$ \\
SunOS 5.7 & gcc 3.1 \\
Windows 2000/XP with Cygwin & gcc $2.95 .3 / 3 . x$ \\
\hline
\end{tabular}

environment to perform inversions with the Optimal Estimation Method (OEM), and to produce sets of spectra to test the inversions, using ARTS as a calculating engine. AMI and Qpack are described separately by Eriksson et al. [this issue].

For IDL there exists a set of interface routines, called AII (ARTS IDL Interface), that can be used to read and write ARTS data files. The AII package is distributed together with ARTS. Finally, there exists a package called arts-data, containing atmospheric states and spectral line catalogues in formats suitable for ARTS. At the moment, the atmospheric scenarios included are CIRA86 [6], FASCOD [7], REFMOD $99,{ }^{3}$ as well as a few cloud test cases. Spectral line catalogues included are MYTRAN [9] and ARTSCAT, which is a converted version of the Verdandi catalogue [10]. For more information about the use of spectral line catalogues see Section 3.2.3.

\subsection{Basic radiative transfer equation}

Under the conditions defined in Section 2.2, the radiative transfer through the atmosphere can be described by a simple differential equation for the specific intensity $I$. With $I$ we mean the power travelling in a given direction, per unit area, per unit solid angle, and per unit frequency interval (see also Section 4.3 about different units of $I$ ). The applicable simplified form of the radiative transfer equation is

$$
\frac{\mathrm{d} I(v, s)}{\mathrm{d} s}=-\alpha(v, s) I(v, s)+\alpha(v, s) B(v, T(s)),
$$

where $\alpha$ is the absorption coefficient in $1 / \mathrm{m}$, and $B$ is the Planck function. The equation describes the change in $I$, as the radiation travels along a path, where the distance along the path is given by $s$. It should be noted that the equation assumes that the path is known, so the problem to determine the path has to be solved separately, as will be described in Section 4. Eq. (1) is a monochromatic equation, i.e., it is valid independently for each frequency, but not valid for frequency averages. This equation is significantly simpler than the general form of the radiative transfer equation, which is described in detail for example by Sreerekha et al. [11].

\footnotetext{
${ }^{3}$ REFMOD99 is a set of atmospheric scenarios that are widely used by the ground-based infrared observation community, in connection with the retrieval package SFIT [8].
} 
A full radiative transfer calculation requires a sequence of problems to be solved:

(1) calculation of absorption coefficients;

(2) integration of the radiative transfer equation;

(3) calculation of Jacobians.

The next three sections (Sections 3-5) describe how ARTS solves these problems.

\section{Absorption}

\subsection{Definitions}

The absorption coefficient $\alpha$, as defined by Eq. (1), can generally be calculated as a sum of different spectral lines of the different gaseous species, plus some additional terms related to absorption continua:

$$
\begin{aligned}
\alpha\left(v, p, T, x_{1}, \ldots, x_{N}\right)= & \sum_{i=1}^{N} \frac{p x_{i}}{k_{\mathrm{B}} T} \sum_{j=1}^{M_{i}} S_{i j}(T) F\left(\tilde{v}_{i j}, v, p, T, x_{1}, \ldots, x_{N}\right) \\
& +C_{1}\left(v, p, T, x_{1}, \ldots, x_{N}\right)+\cdots+C_{L}\left(v, p, T, x_{1}, \ldots, x_{N}\right),
\end{aligned}
$$

where $v$ is the frequency, $T$ the temperature, $p$ the pressure, and $x_{1} \ldots x_{N}$ are the volume mixing ratios of the various gas species. The index $i$ goes over all $N$ gas species and the index $j$ over all $M_{i}$ spectral lines of each gas species. The $k_{\mathrm{B}}$ in the $p x_{i} /\left(k_{\mathrm{B}} T\right)$ term is Boltzmann's constant, which means that this term is nothing else than the partial density $n_{i}$ of gas species $i$.

The contribution of each spectral line is given by the product of the line intensity $S_{i j}(T)$ and the line shape function $F\left(\tilde{v}_{i j}, \ldots\right)$. The first argument of $F, \tilde{v}_{i j}$, is the line centre frequency, which follows directly from the energy difference of the two states involved in the transition, plus a possible pressure shift. (On pressure shift see for example [12].)

In addition to the line spectrum one has to take into account several continua, $C_{1}$ to $C_{L}$, which are in the general case functions of frequency, pressure, temperature, and gas volume mixing ratios.

\subsection{Explicit line-by-line calculation}

\subsubsection{Line strengths}

According to [13] the line strength $S_{i j}(T)$ of Eq. (2) can be calculated as

$$
S_{i j}(T)=S_{i j}\left(T_{0}\right) \frac{Q_{i}\left(T_{0}\right)}{Q_{i}(T)} \frac{\mathrm{e}^{-L_{i j} /\left(k_{\mathrm{B}} T\right)}-\mathrm{e}^{-U_{i j} /\left(k_{\mathrm{B}} T\right)}}{\mathrm{e}^{-L_{i j} /\left(k_{\mathrm{B}} T_{0}\right)}-\mathrm{e}^{-U_{i j} /\left(k_{\mathrm{B}} T_{0}\right)}} .
$$

Here, $S_{i j}\left(T_{0}\right)$ is the line strength at a reference temperature $T_{0}$, which is obtained from the catalogue. The function $Q_{i}(T)$ is the partition function, more correctly the total internal partition sum as defined for example by Gordy and Cook [14]. The parameters $L_{i j}$ and $U_{i j}$ are the energies of the lower and upper state, respectively. The lower state energy is obtained from the database, 
the upper state energy calculated by $U_{i j}=L_{i j}+h \tilde{v}_{i j}$. The $\mathrm{e}^{-(\ldots) /\left(k_{\mathrm{B}} T_{x}\right)}$ terms reflect the Boltzmann distribution of the energy level population.

Overall, all the equation does is scale the line strength from the reference temperature to a different temperature, using that

$$
S_{i j}(T)=\text { const } \frac{\mathrm{e}^{-L_{i j} /\left(k_{\mathrm{B}} T\right)}-\mathrm{e}^{-U_{i j} /\left(k_{\mathrm{B}} T\right)}}{Q_{i}(T)} .
$$

\subsubsection{Line shape functions}

The line shape function $F\left(\tilde{v}_{i j}, \ldots\right)$ has been defined in Eq. (2). ARTS allows the user to select between different line shapes and line shape combinations. Implemented shape functions are listed in Table 2. On the range of applicability or advantages and disadvantages of the different shape functions see [5]. For a theoretical discussion of line shape issues see for example [15,16].

The concept followed in ARTS is to implement the basic line shape building blocks, then allow the user to create the line shape he or she wants by putting the blocks together. One of the implemented basic line shapes is for example the Lorentz line shape:

$$
F_{\mathrm{L}}\left(v, \tilde{v}_{i j}\right)=\frac{\gamma_{\mathrm{L}}}{\pi} \frac{1}{\left(v-\tilde{v}_{i j}\right)^{2}+\gamma_{\mathrm{L}}^{2}} .
$$

The parameter $\gamma_{\mathrm{L}}$ is the Lorentz line width, which is calculated as

$$
\gamma_{L}\left(p, p_{\mathrm{s}}, T\right)=\gamma_{\mathrm{a}}\left(p-p_{\mathrm{s}}\right)\left(\frac{T_{\gamma}}{T}\right)^{n_{\mathrm{a}}}+\gamma_{\mathrm{s}} p_{\mathrm{s}}\left(\frac{T_{\gamma}}{T}\right)^{n_{\mathrm{s}}},
$$

where $p_{\mathrm{s}}$ is the partial pressure of the species considered, $\gamma_{\mathrm{a}}$ and $\gamma_{\mathrm{s}}$ are the air and the selfbroadening parameters, and $n_{\mathrm{a}}$ and $n_{\mathrm{s}}$ are the temperature exponents for $\gamma_{\mathrm{a}}$ and $\gamma_{\mathrm{s}}$, respectively. The temperature $T_{\gamma}$ is the reference temperature for the broadening parameters.

\begin{tabular}{|c|c|c|}
\hline \multirow[t]{6}{*}{ Shape } & "Doppler" & Doppler line shape (a Gauss function) \\
\hline & "Lorentz" & Lorentz line shape \\
\hline & "Voigt_Kuntz" & Kuntz approximation to the Voigt line shape [19] \\
\hline & "Voigt_Drayson", & Drayson approximation to the Voigt line shape [20] \\
\hline & "Rosenkranz_Voigt_Drayson" & $\begin{array}{l}\text { At high pressure a line shape that accounts for line mixing } \\
\text { [21], at low pressure "Voigt_Drayson" }\end{array}$ \\
\hline & "Rosenkranz_Voigt_Kuntz" & The same, but with "Voigt_Kuntz" at low pressure \\
\hline \multirow[t]{5}{*}{ Prefactor } & "no_norm" & 1 \\
\hline & "linear" & $v / \tilde{v}_{i j}$ \\
\hline & "quadratic" & $\left(v / \tilde{v}_{i j}\right)^{2}$ \\
\hline & "VVH” & $v \tanh \left(h v /\left(2 k_{\mathrm{B}} T\right)\right)$ \\
\hline & & $\overline{\tilde{v}_{i j} \tanh \left(h \tilde{v}_{i j} /\left(2 k_{\mathrm{B}} T\right)\right)}$ \\
\hline \multirow[t]{2}{*}{ Cutoff } & “-1” & No cutoff \\
\hline & $x$ & Cutoff at $x \mathrm{GHz}$ \\
\hline
\end{tabular}

Table 2

ARTS line shape options 
A generalization of the Lorentz line shape particularly for the microwave spectral region is the Van Vleck-Weisskopf line shape [17]

$$
\begin{aligned}
F_{\mathrm{VVW}}\left(v, \tilde{v}_{i j}\right) & =\left(\frac{v}{\tilde{v}_{i j}}\right)^{2} \frac{\gamma_{\mathrm{L}}}{\pi}\left[\frac{1}{\left(v-\tilde{v}_{i j}\right)^{2}+\gamma_{\mathrm{L}}^{2}}+\frac{1}{\left(v+\tilde{v}_{i j}\right)^{2}+\gamma_{\mathrm{L}}^{2}}\right] \\
& =\left(\frac{v}{\tilde{v}_{i j}}\right)^{2}\left[F_{\mathrm{L}}\left(v, \tilde{v}_{i j}\right)+F_{\mathrm{L}}\left(v,-\tilde{v}_{i j}\right)\right] .
\end{aligned}
$$

As indicated by Eq. (7), this can be achieved by the ARTS user by choosing a Lorentz shape, adding for each spectral line the mirror line at the negative line centre frequency, and adding a quadratic prefactor.

Our recommended line shape is to take Voigt_Kuntz as the basic shape, and add mirror lines and quadratic prefactor. The resulting line shape behaves like a Van Vleck-Weisskopf shape at high pressure, and like a Voigt shape at low pressure. This avoids the problem of having to switch line shape at some threshold pressure, as some other radiative transfer models do. Alternatively, the quadratic prefactor can be replaced by a Van Vleck-Huber prefactor, as recommended by Rayer [15], but the difference, at least in the sub-millimeter wave spectral region, is small.

Finally, a cutoff can be applied to the line shape, following the procedure described in [18]. The cutoff is applied in such a way that there is no discontinuity at the cutoff frequency (a baseline value is subtracted). Furthermore, the cutoff is applied separately to both line and mirror line.

\subsubsection{Spectral line catalogues}

For practical applications, spectroscopic parameters are not calculated from scratch, but taken from spectroscopic databases, also called spectral line catalogues. In fact, some parameters, such as pressure broadening parameters, cannot be reliably calculated but have to be taken from measurements.

Two of the most widely known catalogues are JPL [13] and HITRAN [22]. The ARTS program can read both of these, and also catalogues in the format MYTRAN, which was defined in an ESA study [9]. Granting the flexibility to work with different catalogues requires a programinternal line data representation that is catalogue independent. This internal representation for each spectral line contains the parameters listed in Table 3.

The parameters $\mathrm{F}$ and PSF are necessary to determine the line position, taking into account a possible pressure shift. The next three parameters, I0, T_I0, and ELOW are to determine the line intensity as a function of temperature (Eq. (3)). The five parameters, AGAM, SGAM, NAIR, NSELF, and T_GAM are necessary to determine the Lorentz line width (Eq. (6)). (The JPL catalogue does not include pressure broadening parameters, so they are simply set to default values when that catalogue is read.) Next there can be some auxiliary parameters AUX1, AUX2,..., which are used for line overlap coefficients (see [1] for details). The rest of the parameters are not necessary for the calculation of absorption coefficients. The seven parameters DF to DPSF contain error estimates for the various parameters, which are needed in order to estimate the impact of errors in spectroscopic parameters on the retrieval of trace gas concentrations or temperature. Then follow some parameters for quantum numbers, and last some indices indicating the information source. 
Table 3

Spectral line parameters in ARTS

\begin{tabular}{|c|c|c|c|c|}
\hline & Description & Short & Symbol & Unit \\
\hline 1 & Name (e.g., O3-666) & NAME & - & - \\
\hline 2 & Centre frequency & $\mathrm{F}$ & $\tilde{v}_{i j}$ & $\mathrm{~Hz}$ \\
\hline 3 & Pressure shift of $F$ & PSF & - & $\mathrm{Hz} / \mathrm{Pa}$ \\
\hline 4 & Line intensity & I0 & $S_{i j}\left(T_{0}\right)$ & $m^{2} \mathrm{~Hz}$ \\
\hline 5 & Reference temp. for I0 & T_I0 & $T_{0}$ & $\mathrm{~K}$ \\
\hline 6 & Lower state energy & ĒōOW & $L_{i j}$ & $\mathbf{J}$ \\
\hline 7 & Air-broadened width & AGAM & $\gamma_{\mathrm{a}}^{\prime \prime}$ & $\mathrm{Hz} / \mathrm{Pa}$ \\
\hline 8 & Self-broadened width & SGAM & $\gamma_{\mathrm{s}}$ & $\mathrm{Hz} / \mathrm{Pa}$ \\
\hline 9 & AGAM temp. exponent & NAIR & $n_{\mathrm{a}}$ & - \\
\hline 10 & SGAM temp. exponent & NSELF & $n_{\mathrm{s}}$ & - \\
\hline 11 & Ref. temp. for AGAM, SGAM & T_GAM & $T_{\gamma}$ & $\mathrm{K}$ \\
\hline 12 & Number of aux. parameters & N_AUX & - & - \\
\hline 13 & Auxiliary parameter & Ā̄X1 & - & - \\
\hline 14 & $\ldots$ & & - & \\
\hline 15 & Error for $\mathrm{F}$ & DF & - & $\mathrm{Hz}$ \\
\hline 16 & Error for $\mathrm{I} 0$ & DI0 & - & $\%$ \\
\hline 17 & Error for AGAM & DAGAM & - & $\%$ \\
\hline 18 & Error for SGAM & DSGAM & - & $\%$ \\
\hline 19 & Error for NAIR & DNAIR & - & $\%$ \\
\hline 20 & Error for NSELF & DNSELF & - & $\%$ \\
\hline 21 & Error for PSF & DPSF & - & $\%$ \\
\hline 22 & Quantum number code & QCODE & - & - \\
\hline 23 & Lower state quanta & QLOWER & - & - \\
\hline 24 & Upper state quanta & QUPPER & - & - \\
\hline 25 & Source of $F$ & IF & - & - \\
\hline 26 & Source of I0 & II0 & - & - \\
\hline 27 & Source of line width variables & ILW & - & - \\
\hline 28 & Source of pressure shift & IPSF & - & - \\
\hline 29 & Source of auxiliary parameters & IAUX & - & - \\
\hline
\end{tabular}

Note: The line intensity refers to the isotope directly, as in JPL, i.e., it does not include the isotopic ratio, as in HITRAN.

The auxiliary parameters can be used to store information that is only used by a few special species, such as line overlap parameters.

Of course, ARTS can also read line catalogues in its own native format. The content of the line file, including units, then corresponds directly to Table 3. The format is described in more detail in [1] Two other important spectroscopic data bases are the SAO catalogue [45] and the GEISA catalogue [46,47]. The former has the same format as HITRAN, thus it should be possible to use it with ARTS, but this option has not yet been tested. The latter, GEISA, has its own format and can thus currently not be used directly with ARTS. However, it would be simple to add also GEISA reading routines. For the time being, a conversion tool that converts the GEISA format to the HITRAN format can be used. It is available from the maintainers of GEISA upon request. 


\subsubsection{Species data}

Since ARTS is not limited to a particular spectroscopic database, species-specific information, such as molecular masses, which are needed to calculate absorption, have to be kept in a central place and in a general format. This species database stores also the tags that are used by different catalogues to identify species and isotope. Thus, it is the central species database that makes the use of different catalogues possible.

Table 4 lists the species/isotopes known to ARTS, as well as the corresponding MYTRAN, HITRAN, and JPL tag numbers. The isotope names are constructed by concatenating the last digits of the atomic weight of the involved atoms, similar to the naming convention in HITRAN. This allows the distinction between "O3-668" (asymmetric $\mathrm{O}_{3}-18$ ) and "O3-686" (symmetric $\mathrm{O}_{3}$ 18).

For each isotope of each species, we store the isotopic ratio, the molecular mass, and some coefficients for the calculation of partition functions. The coefficients were taken from the TIPS program [23], for the species and isotopes covered by TIPS. For a few species not covered by TIPS, such as OClO, coefficients were obtained by making fits to the partition function values that are tabulated along with the 2000 edition of the JPL catalogue. The fits additionally took into account information on vibrational modes from [24,21], where such information was available. Table 4 indicates also the source of the partition function data, where ' $T$ ' means TIPS, ' $J$ ' means JPL, and 'M' means that the value was simply taken from the main isotope. The ARTS User Guide [5] contains detailed information on the procedure that was applied to compare and merge the different partition function data sources.

The spectroscopic databases are changing continuously. The species list in ARTS is almost up to date with the HITRAN list described in [22], with the only exception that the 623 isotope of OCS is missing, as well as the species $\mathrm{HOBr}$ and $\mathrm{C}_{2} \mathrm{H}_{4}$. We plan to add these species in the near future. Furthermore, the available partition function data are also changing continuously. We plan to update the ARTS coefficients to the latest version of TIPS [25], also in the near future.

Great care was taken in designing the species selection mechanism in the ARTS control file. One pseudo-species, i.e., the entity for which absorption coefficients (and Jacobians) can be calculated, can consist of one or all isotopes of a molecular species, or even just a few selected lines. Combinations are also possible. Table 5 gives some examples.

\subsection{Continua}

As stated in Eq. (2), some molecular species show non-resonant continuum absorption in addition to the resonant line absorption. This term varies only slowly with frequency, so slowly that it can be easily distinguished from the line absorption. However, it should be stressed that only the total absorption of a species can be measured, so that the exact distinction between line spectrum and continuum may vary from model to model. Generally, we use continuum terms to bring line by line models into agreement with experimental data of total absorption. The exact magnitude of the continuum terms will thus depend on the details of the line by line model, for example the assumed line shapes, including possible line shape cutoffs.

The continuum absorption models implemented in ARTS are for water vapour $\left(\mathrm{H}_{2} \mathrm{O}\right)$, oxygen $\left(\mathrm{O}_{2}\right)$, nitrogen $\left(\mathrm{N}_{2}\right)$, and carbon dioxide $\left(\mathrm{CO}_{2}\right)$. A summary of the implemented models is given in 
Table 4

Species and isotopes implemented in ARTS

\begin{tabular}{|c|c|c|c|c|c|}
\hline $\begin{array}{l}\text { ARTS } \\
\text { name }\end{array}$ & $\begin{array}{l}\text { ARTS } \\
\text { isot. }\end{array}$ & $\begin{array}{l}\text { Q } \\
\text { src. }\end{array}$ & $\begin{array}{l}\text { MYT. } \\
\text { tag }\end{array}$ & $\begin{array}{l}\text { HIT. } \\
\text { tag }\end{array}$ & $\begin{array}{l}\text { JPL } \\
\text { tags }\end{array}$ \\
\hline \multirow[t]{7}{*}{$\mathrm{H} 2 \mathrm{O}$} & 161 & $\mathrm{~T}$ & 11 & 11 & 18003,18005 \\
\hline & 181 & $\mathrm{~T}$ & 12 & 12 & 20003 \\
\hline & 171 & $\mathrm{~T}$ & 13 & 13 & 19003 \\
\hline & 162 & $\mathrm{~T}$ & 14 & 14 & 19002 \\
\hline & 182 & $\mathrm{~J}$ & - & 15 & 21001 \\
\hline & 172 & M & - & 16 & - \\
\hline & 262 & $\mathrm{~J}$ & - & - & 20001 \\
\hline \multirow[t]{8}{*}{$\mathrm{CO} 2$} & 626 & $\mathrm{~T}$ & 21 & 21 & - \\
\hline & 636 & $\mathrm{~T}$ & 22 & 22 & - \\
\hline & 628 & $\mathrm{~T}$ & 23 & 23 & 46013 \\
\hline & 627 & $\mathrm{~T}$ & 24 & 24 & 45012 \\
\hline & 638 & $\mathrm{~T}$ & 25 & 25 & - \\
\hline & 637 & $\mathrm{~T}$ & 26 & 26 & - \\
\hline & 828 & $\mathrm{~T}$ & 27 & 27 & - \\
\hline & 728 & $\mathrm{~T}$ & 28 & 28 & - \\
\hline \multirow[t]{5}{*}{$\mathrm{O} 3$} & 666 & $\mathrm{~T}$ & 31 & 31 & $48004,48005,48006,48007,48008$ \\
\hline & 668 & $\mathrm{~T}$ & 32 & 32 & 50004,50006 \\
\hline & 686 & $\mathrm{~T}$ & 33 & 33 & 50003,50005 \\
\hline & 667 & $\mathrm{~T}$ & 34 & 34 & 49002 \\
\hline & 676 & $\mathrm{~T}$ & 35 & 35 & 49001 \\
\hline \multirow[t]{5}{*}{$\mathrm{N} 2 \mathrm{O}$} & 446 & $\mathrm{~T}$ & 41 & 41 & $44004,44009,44012$ \\
\hline & 456 & $\mathrm{~T}$ & 42 & 42 & 45007 \\
\hline & 546 & $\mathrm{~T}$ & 43 & 43 & 45008 \\
\hline & 448 & $\mathrm{~T}$ & 44 & 44 & 46007 \\
\hline & 447 & $\mathrm{~T}$ & - & 45 & - \\
\hline \multirow[t]{6}{*}{$\mathrm{CO}$} & 26 & $\mathrm{~T}$ & 51 & 51 & 28001 \\
\hline & 36 & $\mathrm{~T}$ & 52 & 52 & 29001 \\
\hline & 28 & $\mathrm{~T}$ & 53 & 53 & 30001 \\
\hline & 27 & $\mathrm{~T}$ & - & 54 & 29006 \\
\hline & 38 & $\mathrm{~T}$ & - & 55 & - \\
\hline & 37 & $\mathrm{~T}$ & - & 56 & - \\
\hline \multirow[t]{3}{*}{$\mathrm{CH} 4$} & 211 & $\mathrm{~T}$ & - & 61 & - \\
\hline & 311 & $\mathrm{~T}$ & - & 62 & - \\
\hline & 212 & $\mathrm{~T}$ & - & 63 & 17003 \\
\hline \multirow[t]{3}{*}{$\mathrm{O} 2$} & 66 & $\mathrm{~T}$ & 71 & 71 & 32001,32002 \\
\hline & 68 & $\mathrm{~T}$ & 72 & 72 & 34001 \\
\hline & 67 & $\mathrm{~T}$ & 73 & 73 & 33002 \\
\hline \multirow[t]{2}{*}{ NO } & 46 & $\mathrm{~T}$ & 81 & 81 & 30008 \\
\hline & 56 & $\mathrm{~T}$ & - & 82 & - \\
\hline
\end{tabular}


Table 4 (continued)

\begin{tabular}{|c|c|c|c|c|c|}
\hline $\begin{array}{l}\text { ARTS } \\
\text { name }\end{array}$ & $\begin{array}{l}\text { ARTS } \\
\text { isot. }\end{array}$ & $\begin{array}{l}\mathrm{Q} \\
\text { src. }\end{array}$ & $\begin{array}{l}\text { MYT. } \\
\text { tag }\end{array}$ & $\begin{array}{l}\text { HIT. } \\
\text { tag }\end{array}$ & $\begin{array}{l}\text { JPL } \\
\text { tags }\end{array}$ \\
\hline & 48 & $\mathrm{~T}$ & - & 83 & - \\
\hline \multirow[t]{4}{*}{$\mathrm{SO} 2$} & 626 & $\mathrm{~T}$ & 91 & 91 & 64002,64005 \\
\hline & 646 & $\mathrm{~T}$ & 92 & 92 & 66002 \\
\hline & 636 & $\mathrm{~J}$ & 93 & - & 65001 \\
\hline & 628 & $\mathbf{J}$ & 94 & - & 66004 \\
\hline $\mathrm{NO} 2$ & 646 & $\mathrm{~T}$ & 101 & 101 & 46006 \\
\hline \multirow[t]{3}{*}{ NH3 } & 4111 & $\mathrm{~T}$ & 111 & 111 & 17002,17004 \\
\hline & 5111 & $\mathrm{~T}$ & 112 & 112 & 18002 \\
\hline & 4112 & $\mathrm{~J}$ & - & - & 18004 \\
\hline HNO3 & 146 & $\mathbf{J}$ & 121 & 121 & $63001,63002,63003,63004,63005,63006$ \\
\hline \multirow[t]{3}{*}{$\mathrm{OH}$} & 61 & $\mathrm{~T}$ & 131 & 131 & 17001 \\
\hline & 81 & $\mathrm{~T}$ & 132 & 132 & 19001 \\
\hline & 62 & $\mathrm{~T}$ & 133 & 133 & 18001 \\
\hline \multirow[t]{2}{*}{$\mathrm{HF}$} & 19 & $\mathrm{~T}$ & 141 & 141 & 20002 \\
\hline & 29 & $\mathrm{~J}$ & - & - & 21002 \\
\hline \multirow[t]{4}{*}{$\mathrm{HCl}$} & 15 & $\mathrm{~T}$ & 151 & 151 & 36001 \\
\hline & 17 & $\mathrm{~T}$ & 152 & 152 & 38001 \\
\hline & 25 & $\mathbf{J}$ & - & - & 37001 \\
\hline & 27 & $\mathrm{~J}$ & - & - & 39004 \\
\hline \multirow[t]{2}{*}{$\mathrm{HBr}$} & 19 & $\mathrm{~T}$ & 161 & 161 & 80001 \\
\hline & 11 & $\mathrm{~T}$ & 162 & 162 & 82001 \\
\hline $\mathrm{HI}$ & 17 & $\mathrm{~T}$ & - & 171 & - \\
\hline \multirow[t]{2}{*}{$\mathrm{ClO}$} & 56 & $\mathrm{~T}$ & 181 & 181 & 51002,51003 \\
\hline & 76 & $\mathrm{~T}$ & 182 & 182 & 53002,53006 \\
\hline \multirow[t]{5}{*}{ OCS } & 622 & $\mathrm{~T}$ & 191 & 191 & 60001 \\
\hline & 624 & $\mathrm{~T}$ & 192 & 192 & 62001 \\
\hline & 632 & $\mathrm{~T}$ & 193 & 193 & 61001 \\
\hline & 623 & $\mathrm{~T}$ & 194 & 194 & - \\
\hline & 822 & $\mathrm{~T}$ & 195 & 195 & 62002 \\
\hline \multirow[t]{5}{*}{$\mathrm{H} 2 \mathrm{CO}$} & 1126 & $\mathrm{~T}$ & 201 & 201 & 30004 \\
\hline & 1136 & $\mathrm{~T}$ & 202 & 202 & 31002 \\
\hline & 1128 & $\mathrm{~T}$ & 203 & 203 & 32004 \\
\hline & 1226 & $\mathbf{J}$ & - & - & 31003 \\
\hline & 2226 & $\mathrm{~J}$ & - & - & 32006 \\
\hline
\end{tabular}


Table 4 (continued)

\begin{tabular}{|c|c|c|c|c|c|}
\hline $\begin{array}{l}\text { ARTS } \\
\text { name }\end{array}$ & $\begin{array}{l}\text { ARTS } \\
\text { isot. }\end{array}$ & $\begin{array}{l}\text { Q } \\
\text { src. }\end{array}$ & $\begin{array}{l}\text { MYT. } \\
\text { tag }\end{array}$ & $\begin{array}{l}\text { HIT. } \\
\text { tag }\end{array}$ & $\begin{array}{l}\text { JPL } \\
\text { tags }\end{array}$ \\
\hline $\mathrm{HOCl}$ & $\begin{array}{l}165 \\
167\end{array}$ & $\begin{array}{l}\mathrm{T} \\
\mathrm{T}\end{array}$ & $\begin{array}{l}211 \\
212\end{array}$ & $\begin{array}{l}211 \\
212\end{array}$ & $\begin{array}{l}52006 \\
54005\end{array}$ \\
\hline N2 & 44 & $\mathrm{~T}$ & - & 221 & - \\
\hline $\mathrm{HCN}$ & $\begin{array}{l}124 \\
134 \\
125 \\
224\end{array}$ & $\begin{array}{l}\mathrm{T} \\
\mathrm{T} \\
\mathrm{T} \\
\mathrm{J}\end{array}$ & $\begin{array}{l}231 \\
232 \\
233 \\
-\end{array}$ & $\begin{array}{l}231 \\
232 \\
233 \\
-\end{array}$ & $\begin{array}{c}27001,27003 \\
28002 \\
28003 \\
28004\end{array}$ \\
\hline $\mathrm{CH} 3 \mathrm{Cl}$ & $\begin{array}{l}215 \\
217\end{array}$ & $\begin{array}{l}\mathrm{T} \\
\mathrm{T}\end{array}$ & $\begin{array}{l}241 \\
242\end{array}$ & $\begin{array}{l}241 \\
242\end{array}$ & $\begin{array}{l}50007 \\
52009\end{array}$ \\
\hline $\mathrm{H} 2 \mathrm{O} 2$ & 1661 & $\mathrm{~T}$ & 251 & 251 & 34004 \\
\hline $\mathrm{C} 2 \mathrm{H} 2$ & $\begin{array}{l}1221 \\
1231\end{array}$ & $\begin{array}{l}\mathrm{T} \\
\mathrm{T}\end{array}$ & - & $\begin{array}{l}261 \\
262\end{array}$ & - \\
\hline C2H6 & 1221 & $\mathrm{~T}$ & - & 271 & - \\
\hline PH3 & 1111 & $\mathrm{~T}$ & 281 & 281 & 34003 \\
\hline COF2 & 269 & $\mathrm{~T}$ & 291 & 291 & 66001 \\
\hline SF6 & 29 & $\mathrm{~T}$ & - & 301 & - \\
\hline $\mathrm{H} 2 \mathrm{~S}$ & $\begin{array}{l}121 \\
141 \\
131 \\
122\end{array}$ & $\begin{array}{l}\mathrm{T} \\
\mathrm{T} \\
\mathrm{T} \\
\mathrm{J}\end{array}$ & $\begin{array}{l}311 \\
- \\
-\end{array}$ & $\begin{array}{l}311 \\
312 \\
313 \\
-\end{array}$ & $\begin{array}{c}34002 \\
- \\
- \\
35001\end{array}$ \\
\hline $\mathrm{HCOOH}$ & $\begin{array}{l}1261 \\
1361 \\
2261 \\
1262\end{array}$ & $\begin{array}{l}\mathrm{T} \\
\mathrm{J} \\
\mathrm{J} \\
\mathrm{J}\end{array}$ & $\begin{array}{l}321 \\
- \\
-\end{array}$ & $\begin{array}{l}321 \\
- \\
-\end{array}$ & $\begin{array}{l}46005 \\
47002 \\
47003 \\
47004\end{array}$ \\
\hline $\mathrm{HO} 2$ & 166 & $\mathrm{~T}$ & 331 & 331 & 33001 \\
\hline $\mathrm{O}$ & 6 & $\mathrm{~T}$ & 341 & 341 & 16001 \\
\hline $\mathrm{ClONO} 2$ & $\begin{array}{l}5646 \\
7646\end{array}$ & $\begin{array}{l}\mathrm{J} \\
\mathrm{J}\end{array}$ & $\begin{array}{l}351 \\
352\end{array}$ & $\begin{array}{l}351 \\
352\end{array}$ & $\begin{array}{l}97002 \\
99001\end{array}$ \\
\hline $\mathrm{NO}+$ & 46 & $\mathrm{~T}$ & - & 361 & 30011 \\
\hline $\mathrm{OClO}$ & $\begin{array}{l}656 \\
676\end{array}$ & $\begin{array}{l}\mathbf{J} \\
\mathbf{J}\end{array}$ & $\begin{array}{l}431 \\
432\end{array}$ & - & $\begin{array}{l}67001 \\
69001\end{array}$ \\
\hline
\end{tabular}


Table 4 (continued)

\begin{tabular}{|c|c|c|c|c|c|}
\hline $\begin{array}{l}\text { ARTS } \\
\text { name }\end{array}$ & $\begin{array}{l}\text { ARTS } \\
\text { isot. }\end{array}$ & $\begin{array}{l}\text { Q } \\
\text { src. }\end{array}$ & $\begin{array}{l}\text { MYT. } \\
\text { tag }\end{array}$ & $\begin{array}{l}\text { HIT. } \\
\text { tag }\end{array}$ & $\begin{array}{l}\text { JPL } \\
\text { tags }\end{array}$ \\
\hline $\mathrm{BrO}$ & $\begin{array}{l}96 \\
16\end{array}$ & $\begin{array}{l}\mathrm{J} \\
\mathrm{J}\end{array}$ & $\begin{array}{l}401 \\
402\end{array}$ & - & $\begin{array}{l}95001 \\
97001\end{array}$ \\
\hline $\mathrm{H} 2 \mathrm{SO} 4$ & 126 & $\mathrm{~J}$ & 481 & - & 98001 \\
\hline $\mathrm{Cl} 2 \mathrm{O} 2$ & $\begin{array}{l}565 \\
765\end{array}$ & $\begin{array}{l}\mathrm{J} \\
\mathrm{J}\end{array}$ & $\begin{array}{l}491 \\
492\end{array}$ & - & $\begin{array}{l}102001 \\
104001\end{array}$ \\
\hline $\mathrm{HOBr}$ & $\begin{array}{l}169 \\
161\end{array}$ & $\begin{array}{l}\mathrm{J} \\
\mathrm{J}\end{array}$ & $\begin{array}{l}371 \\
372\end{array}$ & $\begin{array}{l}371 \\
372\end{array}$ & $\begin{array}{l}96001 \\
98002\end{array}$ \\
\hline $\mathrm{C} 2 \mathrm{H} 4$ & $\begin{array}{l}221 \\
231\end{array}$ & $\begin{array}{l}\mathrm{T} \\
\mathrm{T}\end{array}$ & - & $\begin{array}{l}381 \\
382\end{array}$ & - \\
\hline $\mathrm{CH} 3 \mathrm{CN}$ & $\begin{array}{l}211124 \\
311124 \\
211134 \\
211125 \\
211224\end{array}$ & $\begin{array}{l}\mathrm{J} \\
\mathrm{J} \\
\mathrm{J} \\
\mathrm{J} \\
\mathrm{J}\end{array}$ & $\begin{array}{l}- \\
- \\
- \\
-\end{array}$ & $\begin{array}{l}- \\
- \\
- \\
-\end{array}$ & $\begin{array}{l}41001 \\
42006 \\
42007 \\
42007 \\
42008\end{array}$ \\
\hline $\mathrm{HNC}$ & $\begin{array}{l}142 \\
143 \\
152 \\
242\end{array}$ & $\begin{array}{l}\mathrm{J} \\
\mathrm{J} \\
\mathrm{J} \\
\mathrm{J}\end{array}$ & $\begin{array}{l}- \\
- \\
-\end{array}$ & $\begin{array}{l}- \\
- \\
-\end{array}$ & $\begin{array}{l}27002 \\
28005 \\
28006 \\
28007\end{array}$ \\
\hline
\end{tabular}

Table 5

Examples of ARTS pseudo-species, for which absorption coefficients and radiative transfer Jacobians can be calculated

\begin{tabular}{ll}
\hline "O3" & All ozone lines \\
"O3-668" & All ozone lines of the 16-16-18 isotope \\
"O3-668,O3-686" & All ozone lines of the 16-16-18 isotope and the 16-18-16 isotope \\
"H2O-161-180e9-185e9" & All water vapour lines of the main isotope between 180 \\
& and $185 \mathrm{GHz}$, i.e., just the $183 \mathrm{GHz}$ line
\end{tabular}

Table 6. Since these molecules have various permanent electric or magnetic multipoles, the physical explanations for the continuum absorption is different in each case.

Water vapour has a strong electric dipole moment and therefore has a wealth of rotational transitions in the microwave up to the sub-millimetre range. One explanation for the $\mathrm{H}_{2} \mathrm{O}$ continuum absorption is the inadequate formulation of the far wings of the spectral lines. The usually employed Van Vleck-Weisskopf line shape [17] is derived under the impact 
Table 6

Implemented continua ('cont.') and complete absorption models ('full')

\begin{tabular}{|c|c|c|c|c|c|c|c|c|}
\hline Name \& Ref. & $\begin{array}{l}\mathrm{H}_{2} \mathrm{O} \\
\text { cont. }\end{array}$ & $\begin{array}{l}\mathrm{H}_{2} \mathrm{O} \\
\text { full }\end{array}$ & $\begin{array}{l}\mathrm{O}_{2} \\
\text { cont. }\end{array}$ & $\begin{array}{l}\mathrm{O}_{2} \\
\text { full }\end{array}$ & $\begin{array}{l}\mathrm{N}_{2} \\
\text { cont. }\end{array}$ & $\begin{array}{l}\mathrm{CO}_{2} \\
\text { cont }\end{array}$ & $\begin{array}{l}\text { Liquid } \\
\text { cloud }\end{array}$ & Rain \\
\hline PWR88 [26] & & & $x$ & $x$ & & & & \\
\hline PWR93 [21] & $x$ & $x$ & $x$ & $x$ & $x$ & $x$ & & \\
\hline PWR98 [27] & $x$ & $x$ & $x$ & $x$ & & & & \\
\hline CruzPo198 [28] & $x$ & $x$ & & & & & & \\
\hline MPM85 [29] & & & $x$ & $x$ & & & & \\
\hline MPM87 [30] & $x$ & $x$ & $x$ & $x$ & & & & \\
\hline MPM89 [31] & $x$ & $x$ & $x$ & $x$ & & & & \\
\hline MPM92 [32] & & & $x$ & $x$ & & & & \\
\hline MPM93 [33] & $x$ & $x$ & $x$ & $x$ & $x$ & & $x$ & $x$ \\
\hline CKD2.4.1 [18] & $x$ & & & & & & & \\
\hline BF86 [34] & & & & & $x$ & & & \\
\hline MT02 [35] & $x$ & & & & & & & \\
\hline AAM02 [36] & & $x$ & & & & & & \\
\hline
\end{tabular}

approximation, and hence only valid in the near wing zone. Other explanations are (see [21] for details) far wing contribution from far-infrared water vapour lines, collision-induced absorption, and water polymer absorption. At present one cannot decide which of these explanations is the correct one, probably all of them play a more or less important role, depending on the frequency range. Luckily, the water vapour continuum can be adequately described by very simple parameterisations $[37,27,36]$ for practical purposes.

Molecular oxygen is one of the few species in the Earth's atmosphere with a permanent magnetic dipole moment. The aligned spins of the two valence electrons lead to a ${ }^{3} \sum$ ground state. According to the selection rules for magnetic dipole transitions, transitions with resonance frequency equal to zero are allowed. Such transitions have a characteristic Debye line shape function, which results in a very slow frequency dependence [21].

Molecular nitrogen has an electric quadrupole moment of modest magnitude. For the frequency range below $1 \mathrm{THz}$ the collision-induced rotation absorption band [38] is most important. The band centre is around $3 \mathrm{THz}$, at $1 \mathrm{THz}$ the band strength is approximately $\frac{1}{6}$ of the maximum value (see Fig. 5.2 of [38]). During collisions, the electric field of the quadrupole moment of one molecule induces a dipole moment in the other molecule. This allows rotational transitions according to the electric quadrupole selection rules, $|\Delta J|=0.2$ (see [21] for details). A similar collision-induced absorption band, with a maximum at $1.5 \mathrm{THz}$, is shown by carbon dioxide (Fig. 5.10 of [38]), but this is negligible in the Earth's atmosphere. Characteristic for collision-induced absorption is the dependency on the square of the molecular density.

It should be stressed that not all combinations of continuum and line by line model make sense, in particular in the case of water vapour, since continua depend on the details of the line by line calculation for which they were derived. The user simply has to know what she or he is doing. Otherwise, it may be safer to use one of the complete absorption models for water vapour and oxygen, which are also available in ARTS. They are briefly described in the next section. 


\subsection{Complete absorption models}

Instead of making an explicit line by line calculation and then adding a continuum term, the user of ARTS may also use some complete absorption models. The available options are also listed in Table 6.

All these models internally contain a collection of spectral lines, as well as matching continua. There are two advantage of using a complete absorption model: Firstly, the computation may be faster, because some of the models use only a reduced line list. Secondly, the user has the guarantee that the total absorption of that species has the value that was intended by the developer of the model.

On the other hand, there is no guarantee that the complete models contain all lines that might be of interest for some particular narrow frequency region, due to the often reduced line database. Generally, they have been developed mostly to correctly reproduce the large-scale features in water vapour and oxygen absorption.

\subsection{Hydrometeor absorption models}

The crude cloud droplet and rain model of Liebe et al. [33] has also been implemented, largely for the benefit of a study of the planned radio occultation instrument ACE + [39]. It has to be stressed, though, that hydrometeors may introduce other effects than just adding absorption. In particular, ice crystals in cirrus clouds, and also rain, act as scatterers, and thus require a completely different radiative transfer scheme. Such a scheme has been implemented in the 1-1 branch of ARTS, following the concept of [11], but extended to spherical geometry and a full Stokes vector treatment.

For the simulation of transmission measurements (neglecting the thermal source term in Eq. (1)) the models of Liebe should be adequate, since they actually predict extinction due to absorption and scattering, rather than just absorption. The cloud droplet model may also be applicable to emission measurements, but the rain model certainly is not, since scattering effects contribute significantly to the rain extinction.

\section{Radiative transfer}

This section deals with the solution of the radiative transfer problem described by Eq. (1). That is, we assume no scattering, no polarisation effects, and local thermodynamic equilibrium. The limitation to cases with a spherical symmetry (Section 2.2) is also relevant for this section.

\subsection{Propagation path calculations}

The first task in solving the radiative transfer problem is to determine the propagation path, i.e., the path through the atmosphere travelled by the radiation reaching the sensor. Refraction affects the radiative transfer in several ways. The most notable effect is that for limb sounding the tangent point is displaced vertically. The tangent point is displaced downwards compared to the pure geometrical case (for a fixed observation direction), therefore inclusion of refraction in general 
gives higher intensities. There is also a horizontal displacement of the tangent point, but that is not important for a spherically symmetric atmosphere, except for the fact that the distance traversed through the atmospheric layers is changed.

As the refractive index has a frequency dependency (see e.g. [31]), the atmosphere is in principle dispersive. Each frequency component has its own propagation path. For measurements using frequencies below $1000 \mathrm{GHz}$, notable dispersion will only take place for limb sounding into the troposphere, ${ }^{4}$ but only around a few water vapour transitions [2], having in common a very strong absorption. The atmosphere is totally opaque at lower altitudes around these transitions and the propagation path through the troposphere does not influence the radiance seen by a sensor placed in space. Thus, dispersion can be neglected for the discussed frequency range, and is in fact neglected in ARTS. The (non-dispersive) refractive index $n$ is calculated as following [40]:

$$
n(p, T, e)=1+77.593 \times 10^{-8} \frac{p-e}{T}+e\left(\frac{72 \times 10^{-8}}{T}+\frac{3.754 \times 10^{-3}}{T^{2}}\right),
$$

where $p$ is the total air pressure in $\mathrm{Pa}, T$ the temperature in $\mathrm{K}$ and $e$ the water vapour pressure in $\mathrm{Pa}$.

The propagation path is described by a set of points in the atmosphere, equally spaced in distance along the path. The user sets an upper limit for the distance between the path points. The lowermost altitude of the path is always included among the path points. This is either the position of the sensor, the ground, or the tangent point, depending on sensor position and line-ofsight. The lowermost altitude is also the starting point for the calculations. For cases with the sensor inside the atmosphere and a downward looking geometry (zenith angle above $90^{\circ}$ ), the distance between the path points is adjusted to get an integer number of steps between the lowermost point and the altitude of the sensor. The user-specified distance is used for all other cases. The practical upper limit of the atmosphere is set by the altitude of the uppermost pressure surface.

Propagation paths can be calculated neglecting refraction effects. This results in pure geometrical calculations, handled by the Pythagorean rule and standard trigonometric expressions (see the ARTS user guide [5] for details). The calculations including refraction effects are based on Snell's law for spherical symmetry, stating that the product of the radius, refractive index $n$, and zenith angle $\theta$ is constant along the propagation path (see the user guide, or e.g. [41-43]):

$$
\gamma=\left(R_{\mathrm{e}}+z\right) n(z) \sin (\theta),
$$

where $\gamma$ is the path constant, $R_{\mathrm{e}}$ is the Earth geoid radius, and $z$ the altitude above the geoid. The path constant is determined by the zenith angle of the sensors line-of-sight, and the radius and refractive index at the sensor position. As mentioned above, the calculations start by determining the lowest altitude of the path. For downward observations, the tangent altitude $z_{t}$ is found by the implicit expression

$$
\left(R_{\mathrm{e}}+z_{t}\right) n\left(z_{t}\right)=\gamma .
$$

\footnotetext{
${ }^{4}$ Effects of the ionosphere are neglected here.
} 
The tangent altitude will normally not happen to be on a model grid point, but between two grid points. It is determined practically by linear interpolation in $\gamma^{\prime}(z)=\left(R_{\mathrm{e}}+z\right) n(z)$. If the tangent altitude would be below the surface the sensor sees the ground. In that case the zenith angle of the propagation path at the ground, $\theta_{\mathrm{g}}$, is given by

$$
\theta_{\mathrm{g}}=\sin ^{-1}\left(\frac{\gamma}{\left(R_{\mathrm{e}}+z_{\mathrm{g}}\right) n\left(z_{\mathrm{g}}\right)}\right) \text {. }
$$

The radiative transfer is evaluated along the propagation path, while Eq. (9) is expressed for vertical altitudes. The relationship between a change in vertical altitude and the corresponding distance along the path is here denoted as the geometrical term and it is

$$
g(z)=\frac{\mathrm{d} s}{\mathrm{~d} z}=\frac{1}{\cos (\theta)},
$$

which can be rewritten, using trigonometric identities and Eq. (9), to

$$
g(z)=\frac{\left(R_{\mathrm{e}}+z\right) n(z)}{\sqrt{[}\left(R_{\mathrm{e}}+z\right)^{2} n^{2}(z)-\gamma^{2}} .
$$

If the refractive index is assumed to be constant between altitude $z_{1}$ and $z_{2}$, Eq. (13) gives

$$
\Delta s=\sqrt{[}\left(R_{\mathrm{e}}+z_{2}\right)^{2}-\left(\frac{\gamma}{\bar{n}}\right)^{2}-\sqrt{[]}\left(R_{\mathrm{e}}+z_{1}\right)^{2}-\left(\frac{\gamma}{\bar{n}}\right)^{2},
$$

where $\Delta s$ is the distance along the propagation path between $z_{1}$ and $z_{2}, z_{2}>z_{1}$ and $\bar{n}=\left(n\left(z_{1}\right)+n\left(z_{2}\right)\right) / 2$. Further details of these calculations are given in the ARTS user guide.

The fact that the propagation path calculation starts at the point where the atmosphere has the highest density helps to maintain a high accuracy of the calculations involving refraction. A further advantage is that a symmetry point of the propagation path is obtained for limb observations, and it is sufficient to determine only the path on one side of the lowermost altitude.

\subsection{The radiative transfer equation}

With the assumptions applied for ARTS-1-0-x, the radiative transfer equation (Eq. (1)) can be solved analytically:

$$
I(v)=I_{0}(v) \mathrm{e}^{-\tau(v, 0, l)}+\int_{0}^{l} B(v, T(s)) \alpha(v, s) \mathrm{e}^{-\tau(v, 0, s)} \mathrm{d} s,
$$

where $I_{0}$ is the intensity at the practical starting point of the propagation path, at the distance $l$ from the observation position, and

$$
\tau\left(v, l_{1}, l_{2}\right)=\int_{l_{1}}^{l_{2}} \alpha(v, s) \mathrm{d} s
$$

is the optical depth along (a part of) the propagation path.

Different numerical schemes to solve Eq. (15) exist (see e.g. [43]). In the approach selected here, the radiation is followed along the path from one point to the next, using the expression

$$
I_{q+1}=I_{q} \mathrm{e}^{-\tau_{q}}+\bar{B}_{q}\left(1-\mathrm{e}^{-\tau_{q}}\right),
$$


where

$$
\begin{gathered}
\tau_{q}=\Delta s \frac{\alpha_{q}+\alpha_{q+1}}{2}, \\
\bar{B}_{q}=\frac{B_{q}+B_{q+1}}{2} .
\end{gathered}
$$

$I_{q}$ is the intensity reaching point $q, \alpha_{q+1}$ is the absorption at path point $q+1$, and so on. Eq. (17) is derived under the condition that the Planck function is constant for each step, and can be approximated by the mean of the values at the end points. Absorption variations are assumed to be piecewise linear.

If path point $q$ corresponds to an intersection with the ground, the ground reflection is considered as

$$
I_{q} \leftarrow I_{q}(1-e(v))+e(v) B\left(v, T_{\mathrm{g}}\right),
$$

where $e$ is the ground emission factor and $T_{\mathrm{g}}$ is the temperature of the ground.

For cases when emission is neglected, the program calculates the total optical thickness along the propagation path following Eq. (16). The piecewise linear variation of the absorption along the propagation path, expressed by Eq. (18), is also assumed for these calculations.

\subsection{Radiance units}

The basic unit for the radiance, $I$, is $\mathrm{W} / \mathrm{m}^{2} / \mathrm{sr} / \mathrm{Hz}$. For microwave measurements it is customary to express the radiance in a temperature scale, the brightness temperature $T_{\mathrm{b}}$. The basic idea is that the brightness temperature is the temperature that a blackbody must have to give the same radiance as measured. This is achieved by calculating the brightness temperature by "inverting" the Planck function:

$$
T_{\mathrm{b}}=\frac{h v}{k_{\mathrm{B}}} \frac{1}{\ln \left(2 h v^{3} /\left(c^{2} I\right)+1\right)},
$$

where $h$ is the Planck constant, $k_{\mathrm{B}}$ is the Boltzmann constant, and $c$ is the speed of light. However, as the Planck brightness temperature involves a non-linear scaling between $I$ and $T_{\mathrm{b}}$, it can only be used to display spectra on a temperature scale, not for Jacobians. A linear relationship between $I$ and $T_{\mathrm{b}}$ is obtained by starting with the Rayleigh-Jeans approximation (valid for $h v \ll k_{\mathrm{B}} T$ ) of the Planck function, that inverted gives

$$
T_{\mathrm{b}}=\frac{c^{2}}{2 v^{2} k_{\mathrm{B}}} I \text {. }
$$

As the Rayleigh-Jeans brightness temperature is linearly proportional to the radiance, the unit can be used when performing retrievals, which requires that both spectra and Jacobians are presented in this unit. The deviation between Planck and Rayleigh-Jeans brightness temperatures is smaller than 0.8 and $8 \mathrm{~K}$ at 30 and $300 \mathrm{GHz}$, respectively.

ARTS calculates in radiance units $\left(\mathrm{W} / \mathrm{m}^{2} / \mathrm{sr} / \mathrm{Hz}\right)$ internally. At the end, the user can convert these to Planck brightness temperature or Rayleigh-Jeans brightness temperature, as desired. 


\section{Jacobians}

\subsection{Theory}

The complete forward model, including sensor characteristics, is here expressed as $[44,43]$

$$
\mathbf{y}=F(\mathbf{x})+\varepsilon,
$$

where $\mathbf{y}$ is the vector of measurement data, the vector $\mathbf{x}$ holds all forward model parameters, and $\varepsilon$ is the measurement noise. Following Eriksson et al. [this issue], the forward model is divided into a first part handling atmospheric radiative transfer

$$
\mathbf{i}=F_{r}\left(\mathbf{x}_{r}\right)
$$

and a second part incorporating sensor characteristics and data reduction,

$$
\mathbf{y}=F_{\mathrm{s}}\left(\mathbf{i}, \mathbf{x}_{\mathrm{s}}\right)+\varepsilon,
$$

where $\mathbf{i}$ is a vector holding monochromatic pencil beam spectral values (as given by Eq. (15)) or corresponding optical thicknesses, and the vector $\mathbf{x}$ is divided into two parts, following the division of the forward model. It is assumed here that $F_{\mathrm{s}}$ can be implemented as a multiplication between a matrix $H$ and the vector $\mathbf{i}$ (see Eriksson et al. [this issue] [48] for details):

$$
\mathbf{y}=\mathbf{H i}+\varepsilon .
$$

The partial derivatives of the forward model with respect to the variables that are part of $\mathbf{x}$,

$$
\mathbf{K}=\frac{\partial F}{\partial \mathbf{x}},
$$

are here denoted as the Jacobians, but other names are also common, such as the weighting functions. Sections 5.2 and 5.3 discuss how the Jacobians are calculated practically.

\subsection{Numerical perturbations}

A general and straightforward method to calculate a Jacobian is to disturb the parameter of interest slightly from the nominal value and perform a completely new forward model calculation:

$$
\mathbf{K}_{p}=\frac{F\left(\mathbf{x}+\Delta \mathbf{x}_{p} \mathbf{e}_{p}\right)-F(\mathbf{x})}{\Delta \mathbf{x}_{p}},
$$

where $\mathbf{K}_{p}$ is column $p$ of the $\mathbf{K}$ matrix, $\mathbf{x}$ is the linearisation state for the calculations, $\mathbf{e}_{p}$ is a vector of zeros except for component $p$ that is unity, and $\Delta \mathbf{x}_{p}$ is a small scalar disturbance (but sufficiently large to avoid numerical instabilities).

This calculation approach is used to derive the Jacobians for pointing and frequency offsets, and atmospheric temperatures, when also non-local effects due to the hydrostatic balance are considered. An offset means that all zenith angles of a measurement sequence, or the frequency of all spectrometer channels, are shifted from the nominal value by the same amount.

The perturbation approach is general and simple to implement, but computationally expensive, even though the algorithms in ARTS avoid as far as possible the recalculation of quantities not 
affected by the perturbation. For example, a recalculation of absorption is not needed to determine the pointing offset Jacobian.

\subsection{Analytical expressions}

For some atmospheric variables, such as species abundances, it is possible to derive analytical expressions for the Jacobians. This is advantageous as it results in both faster and more accurate calculations. Only cases with emission are considered here. The Jacobians for pure transmission measurements lead to simpler expressions, which are given in the ARTS user guide.

Under the condition that the variable of interest only influences the local absorption and Planck function, Eqs. (27), (26), (15), and the chain rule give

$$
\mathbf{K}_{p}=\mathbf{H} \frac{\partial \mathbf{i}}{\partial \mathbf{x}_{p}}=\mathbf{H}\left[\frac{\partial \mathbf{i}}{\partial \beta_{p}} \frac{\partial \beta_{p}}{\partial \mathbf{x}_{p}}+\frac{\partial \mathbf{i}}{\partial a_{p}} \frac{\partial a_{p}}{\partial \mathbf{x}_{p}}\right],
$$

where $\beta_{p}$ and $a_{p}$ are the Planck function and total absorption, respectively, at the vertical altitude corresponding to $\mathbf{x}_{p}$. Eq. (29) should be of general validity for species above the tropopause. However, it is only approximately correct if the variable of interest significantly affects the refractive index, and thus the propagation path. This point must be considered for temperature in general and water vapour in the lower troposphere.

The terms $\beta$ and $a$ in Eq. (29) are the Planck function and absorption as a function of altitude, since atmospheric quantities are represented as vertical profiles. However, the radiative transfer is calculated along the propagation path, therefore Eq. (29) has to be expanded one step further to

$$
\mathbf{K}_{p}=\mathbf{H}\left[\frac{\partial \mathbf{i}}{\partial B} \frac{\partial B}{\partial \beta_{p}} \frac{\partial \beta_{p}}{\partial \mathbf{x}_{p}}+\frac{\partial \mathbf{i}}{\partial \alpha} \frac{\partial \alpha}{\partial a_{p}} \frac{\partial a_{p}}{\partial \mathbf{x}_{p}}\right],
$$

where $B$ and $\alpha$ are the Planck function and total absorption, respectively, along the propagation path (cf. Eq. (15)). The Planck function depends only on temperature, therefore $\partial \beta_{p} / \partial \mathbf{x}_{p}=0$ for other quantities; and Eq. (30) can be simplified to

$$
\mathbf{K}_{p}=\mathbf{H} \frac{\partial \mathbf{i}}{\partial \alpha} \frac{\partial \alpha}{\partial a_{p}} \frac{\partial a_{p}}{\partial \mathbf{x}_{p}} .
$$

\subsubsection{Common terms}

The term $\partial \mathbf{i} / \partial \alpha$ expresses how the monochromatic pencil beam radiance is affected by changes of the (total) absorption along the propagation path. The calculation of this term is described in detail in the user guide, including special expressions making use of the symmetry around the tangent point for limb sounding measurements. As an example, the expression for upward observations (the propagation path passes each altitude only once) for points inside the atmosphere is given here. The radiance for this situation can be written as (cf. Eqs. (17)-(19))

$$
I=\left[I_{q+1} \zeta_{q} \zeta_{q-1}+\bar{B}_{q}\left(1-\zeta_{q}\right) \zeta_{q-1}+\bar{B}_{q-1}\left(1-\zeta_{q-1}\right)\right] \Theta_{1}^{q-1}+I_{q-1}^{\prime},
$$


where $q$ indexes the points along the propagation path, with $q=1$ being the path point at the sensor, $I_{q}$ is the radiance reaching point $q$ along the path, $\Theta_{1}^{q}$ is the transmission between point 1 and $q, I_{q}^{\prime}$ is the radiance generated between point $q$ and 1 , and

$$
\zeta_{q}=\exp \left(-\Delta s\left(\alpha_{q}+\alpha_{q+1}\right) / 2\right)
$$

is the transmission between points $q$ and $q+1$. It follows, that

$$
\frac{\partial \zeta_{q}}{\partial \alpha_{q}}=-\frac{\Delta s}{2} \zeta_{q} .
$$

After some re-arrangements, the derivative of the radiance with respect to the absorption at point $q$ can be written as

$$
\frac{\partial \mathbf{i}}{\partial \alpha_{q}}=-\frac{\Delta s}{2}\left[2\left(I_{q+1}-\bar{B}_{q}\right) \zeta_{q}+\bar{B}_{q}-\bar{B}_{q-1}\right] \Theta_{1}^{q} .
$$

The term $\partial \alpha / \partial a_{p}$ in Eqs. (30) and (31) describes the transformation of absorption from the vertical coordinate to distance along the propagation path. The absorption is treated to be a piecewise linear function, both vertically and along the propagation path. The basis functions for a continuous representation of the absorption are thus triangular functions, such as those shown on the left side of Fig. 3. To achieve a consistency between the two absorption terms $a$ and $\alpha$, the following expression should be valid for all positions:

$$
\sum_{i} \mathbf{a}_{i} \phi_{i}^{\mathbf{a}}(z(s))=\sum_{j} \alpha_{j} \phi_{j}^{\alpha}(s),
$$

where $i$ indexes the vertical retrieval altitudes, $z$ is vertical altitude, $\phi^{\mathbf{a}}$ are the vertical basis functions, $j$ indexes the points along the propagation path, and $\phi^{\alpha}$ are the basis functions along the path. However, as can be seen in Fig. 3, $\phi_{i}^{\mathbf{a}}$ as a function of distance along the path $(s)$ is not always a piecewise linear function and can thus not always be fitted perfectly by the basis $\phi_{j}^{\alpha}$.
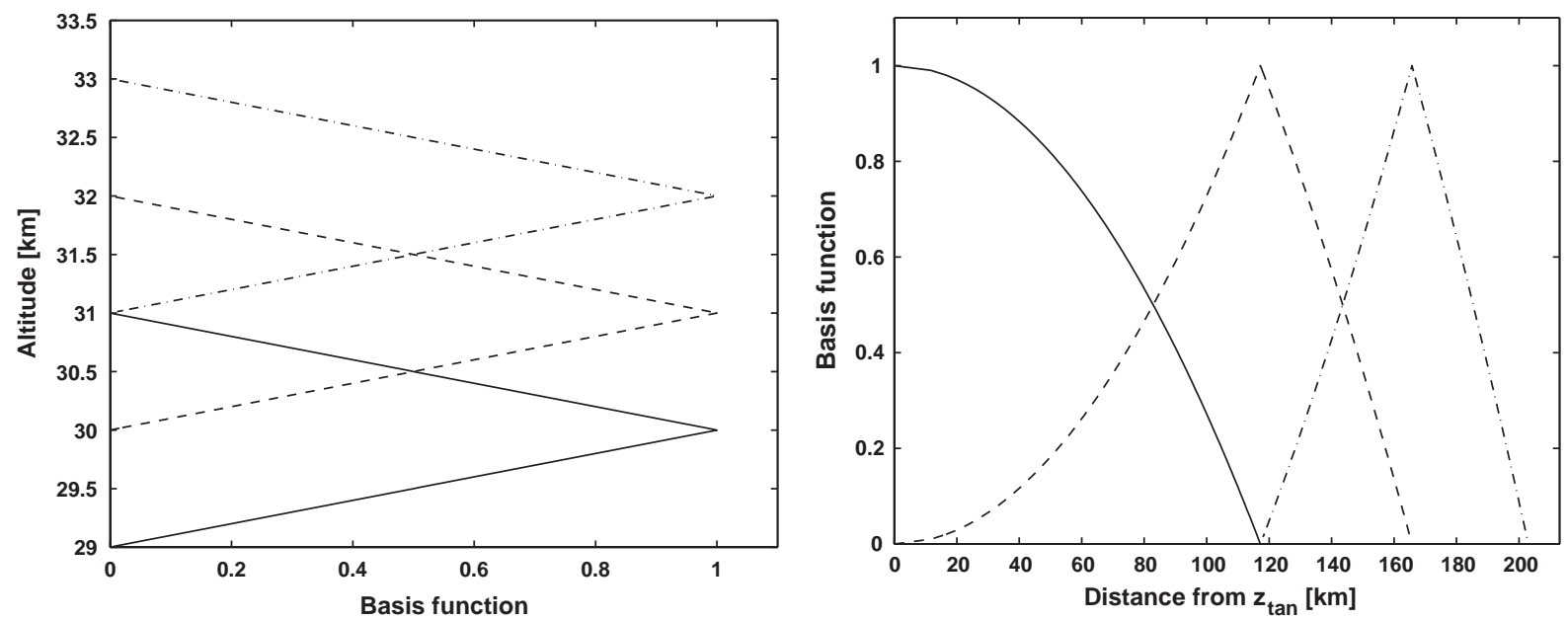

Fig. 3. The left figure shows the basis functions for 30,31 and $32 \mathrm{~km}$ for a vertical grid with a spacing of $1 \mathrm{~km}$. The right figure shows the same functions, but plotted as a function of the distance from the tangent point, where the tangent altitude, $z_{\tan }$, is set to $30 \mathrm{~km}$. 
Hence, some approximation is needed and the most natural choice is to fulfil Eq. (36) at the grid points along the path

$$
\alpha_{q}=\sum_{i} \mathbf{a}_{i} \phi_{i}^{\mathbf{a}}\left(z\left(s_{q}\right)\right)
$$

where $s_{q}$ is the distance along the path for point $q$. This equation follows from the fact that at $s_{j}$ all $\phi_{j}^{\alpha}$ are zero except for $\phi_{q}^{\alpha}$ that is unity. This leads to

$$
\frac{\partial \alpha^{q}}{\partial \mathbf{a}^{p}}=\phi_{\mathbf{a}}^{p}\left(z\left(\mathbf{s}^{q}\right)\right)
$$

Hence, the term $\partial \alpha / \partial \mathbf{a}^{p}$ is determined by the values of $\phi_{\mathbf{a}}^{p}$ at the altitudes corresponding to the grid points of the propagation path.

\subsubsection{Species Jacobians}

The Jacobians for atmospheric species are calculated using Eq. (31). The terms $\partial \mathbf{i} / \partial \alpha$ and $\partial \alpha / \partial a_{p}$ have been discussed above. The remaining term $\partial a_{p} / \partial \mathbf{x}_{p}$ is simply obtained by dividing the absorption of the species with the amount of the species, in the unit selected for the retrievals. Jacobians can be provided for three retrieval units: Number density, volume mixing ratio and relative changes with respect to the normalisation state. For the last unit, the Jacobians correspond to a $100 \%$ increase in species abundance and the term $\partial a_{p} / \partial \mathbf{x}_{p}$ equals the absorption of the species.

The treatment of the term $\partial a_{p} / \partial \mathbf{x}_{p}$ assumes a linear relationship between species abundance and absorption for the species. This assumption is normally valid. The only notable exception is water vapour at low altitudes, where the self-broadening can contribute significantly to the total pressure broadening, leading to a slightly non-linear relationship between species abundance and absorption.

\subsubsection{Continuum absorption Jacobians}

The purpose of these Jacobians is to compensate for imperfect knowledge of absorption of continuum character. That is, absorption without spectral features inside the measured frequency range. The continuum absorption Jacobians are calculated as species Jacobians, except for the calculation of the term $\partial a_{p} / \partial \mathbf{x}_{p}$.

The continuum absorption is represented by a polynomial for each retrieval altitude. The polynomials are characterised by the magnitude of the absorption at a number of points inside the frequency range covered. The points are equally spaced over the frequency range and the number of points follows the selected polynomial order. This approach was selected as it gives the possibility to impose a positive constraint in a straightforward manner. A direct polynomial representation $\left(k=k_{0}+k_{1} v+k_{2} v^{2}+\cdots\right)$ is less favourable regarding this aspect. The term $\partial a_{p} / \partial \mathbf{x}_{p}$ is determined by Lagrange's formula that gives the polynomial of order $N-1$ that passes through $N$ fixed points. Details are given in the user guide.

\subsubsection{Temperature Jacobians}

Jacobians for atmospheric temperatures incorporating effects of hydrostatic equilibrium are calculated by perturbations, as mentioned above. If the temperature is assumed to only affect the local conditions, the Jacobians can be determined by Eq. (30). The partial derivatives will be discussed in the order they appear in that equation. The first term, $\partial \mathbf{i} / \partial B$, expresses how the 
radiance is affected by a change of the Planck function along the propagation path, and it is derived in the same way as $\partial \mathbf{i} / \partial \alpha$. The expression corresponding to Eq. (35) is

$$
\frac{\partial \mathbf{i}}{\partial B_{q}}=\frac{1-\zeta_{q-1} \zeta_{q}}{2} \Theta_{1}^{q-1} .
$$

The second term, $\partial B / \partial \beta_{p}$, is identical to $\partial \alpha / \partial a_{p}$ as temperature and absorption are represented on the same grids (both vertically and along the propagation path). The term $\partial \beta_{p} / \partial \mathbf{x}_{p}$ equals the temperature derivative of the Planck function, $\mathrm{d} B / \mathrm{d} T$, which can be written as [2]

$$
\frac{\mathrm{d} B}{\mathrm{~d} T}=\frac{h v}{k_{\mathrm{B}} T^{2}}\left(\mathrm{e}^{h v / k_{\mathrm{B}} T}-1\right)^{-1} B(v, T) .
$$

The terms $\partial \mathbf{i} / \partial \alpha$ and $\partial \alpha / \partial a_{p}$ have been discussed in Section 5.3.1. The last term $\partial a_{p} / \partial \mathbf{x}_{p}$ represents the temperature partial derivative of the absorption and is calculated numerically by a $+1 \mathrm{~K}$ perturbation. This calculation can be performed for all altitudes in parallel as the absorption depends only on the local temperature.

\subsubsection{Jacobians for spectroscopic parameters}

Jacobians can be calculated for a number of spectroscopic parameters, as defined in Table 3. These are: Line position $\tilde{v}_{i j}$, pressure shift, line intensity $S_{i j}\left(T_{0}\right)$, air broadening parameter $\gamma_{\mathrm{a}}$, selfbroadening parameter $\gamma_{\mathrm{s}}$, air broadening temperature exponent $n_{\mathrm{a}}$, and self-broadening temperature exponent $n_{\mathrm{s}}$.

The terms $\partial \mathbf{i} / \partial \alpha$ and $\partial \alpha / \partial a_{p}$ are calculated analytically as described above. The last term in the chain, $\partial a_{p} / \partial \mathbf{x}_{p}$, in this case the derivative of the absorption coefficient with respect to the spectroscopic parameter, is calculated by perturbation.

\subsubsection{Jacobian for ground emissivity}

The Jacobian for ground emissivity $(e)$ is calculated as

$$
\mathbf{K}_{e}=\mathbf{H} \frac{\partial \mathbf{i}}{\partial e} .
$$

Following the approach in Section 5.3.1 and using Eq. (20), the sensitivity of the monochromatic pencil beam radiances to changes in the ground emission factor is found to be

$$
\frac{\partial \mathbf{i}}{\partial e}=\left[B\left(v, T_{\mathrm{g}}\right)-I_{q}\right] \Theta_{1}^{q},
$$

where $q$ is the path point at the ground. This derivative is of course zero if there is no ground reflection.

\section{Summary and conclusions}

The ARTS is a public domain project which was initiated jointly by the Universities of Bremen, Germany and Chalmers, Sweden. The aim is to create a radiative transfer code that is as general and flexible as possible, while at the same time being portable, and user-friendly. The program itself is written in $\mathrm{C}++$, but is accompanied by interface functions and helper functions in IDL 
and Matlab. The complete source code, along with extensive documentation, is freely available on the Internet, under http://www.sat.uni-bremen.de/arts/.

Absorption coefficients can be calculated line-by-line from an external spectral line catalogue. Besides the catalogue formats of JPL and HITRAN, the model supports the MYTRAN format, which was defined by Bauer et al. [9], and a native catalogue format, as described by Buehler and Eriksson [1]. Modules exist for various line shape functions. Partition functions are calculated from an internal polynomial representation, which is taken from the TIPS program [23] for those species included in TIPS. For the other species, a polynomial fitting the tabulated partition function values of JPL is used.

In addition to the line spectrum, different continuum absorption models can be added, such as the water vapour continua of Liebe and Rosenkranz. As an alternative, the user can choose from several built-in complete absorption models.

The radiative transfer is calculated in equal distance steps along the line of sight, which is advantageous particularly for the limb geometry. All observation positions are possible: On the ground, inside the atmosphere, or on a satellite. Furthermore, for any observation position all viewing directions are possible: Up, limb, or down. Jacobians are calculated analytically as far as possible, using the chain rule. Where perturbations have to be used, the program avoids unnecessary re-calculations as far as possible. Jacobians can be calculated for species concentrations, continuum absorption offsets, atmospheric temperature, instrument pointing, instrument frequency offsets, various spectroscopic parameters, and surface emissivity.

The model has all necessary features to be used in instrument characterisation studies and operational data retrieval. It is particularly useful as forward operator for the inverse model package Qpack, described separately in Eriksson et al. [this issue]. Several papers about the validation of the model against other models and atmospheric data are in preparation.

\section{Outlook}

There are ongoing activities to extend ARTS in several directions. As mentioned in Section 2.2, there is a new ARTS version 1-1-x that can simulate multiple scattering by cirrus clouds. Two different algorithms have been already implemented, a Monte Carlo algorithm, and a successive order of scattering algorithm in discrete ordinates. Since that ARTS version treats the full Stokes vector, it will also be possible to explicitly calculate the effect of Zeeman splitting of oxygen lines in the upper stratosphere.

On the other hand, work is in progress to validate ARTS for the thermal infrared spectral region, and to implement the necessary continua for that spectral region. To that end we are currently participating in the ITWG (International TOVS Working Group) intercomparison of infrared radiative transfer models for the AIRS (Atmospheric Infrared Sounder) instrument.

\section{Acknowledgements}

The authors acknowledge the contribution of all that have helped in the development of the model, or the interfaces to other programs, in particular O. Lemke, N. Koulev, C. Jiménez, and 
W. Haas. We furthermore acknowledge the heritage from the previous radiative transfer model of the Bremen group FORWARD, with credit due particularly to J. Langen, J. Urban, and T. Wehr. Some credit is also due to numerous discussion partners at our annual radiative transfer workshops at Bredbeck, which have turned out to be a great help in the program development. Among many others these are: F. Schreier, Y. Kasai, S. Ochiai, G. Kopp, J. Kaiser, P. Raier, D. Feist, J. Reburn, R. Siddans, F. Merino, and P. Barron. Acknowledgements are also due to S. Korn for updating and checking the data in Table 4. The work was funded by the German Federal Ministry of Education and Research (BMBF), within the AFO2000 project UTH-MOS, Grant 07ATC04, and the DLR project SMILES, Grant 50EE9815. It is a contribution to COST Action 723 'Data Exploitation and Modeling for the Upper Troposphere and Lower Stratosphere'.

\section{References}

[1] Buehler SA, Eriksson P. Atmospheric millimeter and sub-millimeter wave radiative transfer modeling. Berichte aus der Physik, Shaker Verlag GmbH. Aachen; 2000 (ISBN 3-8265-7486-9).

[2] Eriksson P, Merino F, Murtagh D, Baron P, Ricaud P, de la Noë J. Studies for the Odin sub-millimetre radiometer: 1. Radiative transfer and instrument simulation. Can J Phys 2002;80:321-40.

[3] Stallman RM. Free software, free society: selected essays. GNU Press; 2002 (ISBN 1-882114-98-1).

[4] van Heesch D. Doxygen manual. Technical Report, 2003, regularly updated versions available at http:// www.doxygen.org/.

[5] Eriksson P, Buehler SA, Emde C, Sreerekha TR, Melsheimer C, Lemke O. ARTS user guide. Technical Report, University of Bremen, 2003, regularly updated versions available at http://www.sat.uni-bremen.de/arts/.

[6] Fleming EL, Chandra S, Barnett JJ, Corney M. Zonal mean temperature, pressure, zonal wind, and geopotential height as function of latitude. Adv Space Res 1990;10(12):11-59.

[7] Anderson GP, Clough SA, Kneizys FX, Chetwynd JH, Shettle EP. AFGL atmospheric constituent profiles (0-120 km). Technical Report TR-86-0110, AFGL, 1986.

[8] Rinsland CP, et al. Infrared measurements of HF and HCL total column abundances above kitt peak, 1977-1990: seasonal cycles, long-term increases, and comparisons with model calculations. J Geophys Res 1991;96:15523-40.

[9] Bauer A, Birk M, Buehler SA, Colmont J, von Engeln A, Kuenzi K, et al. Study on a spectroscopic data base for millimeter and submillimeter wavelengths, final report. Technical Report, ESTEC Contract No. 11581-95-NL-CN, February 1998.

[10] Eriksson P. The verdandi database. Technical Report, availableonline at http:/www.rss.chalmers.se/gem/ Research/verdandi.html, 2003.

[11] Sreerekha TR, Buehler SA, Emde C. A simple new radiative transfer model for simulating the effect of cirrus clouds in the microwave spectral region. JQSRT 2002;75:611-24.

[12] Pumphrey HC, Buehler SA. Instrumental and spectral parameters: their effect on and measurement by microwave limb sounding of the atmosphere. JQSRT 1999;64(4):421-37.

[13] Pickett HM, Poynter RL, Cohen EA. Submillimeter, millimeter, and microwave spectral line catalogue. Technical Report JPL Publication 80-23, Rev. 3, Jet Propulsion Laboratory, 1992.

[14] Gordy W, Cook R. Microwave molecular spectra. New York: Interscience Publishers; 1970 (ISBN 471931616 ).

[15] Rayer P. The VVH and VVW spectral functions. In: Eriksson P, Buehler SA, editros. Atmospheric millimeter and sub-millimeter wave radiative transfer modeling II. Berlin: Logos Verlag; 2001 (ISBN 3-89722-585-9, ISBN 1615-6862).

[16] Van Vleck JH, Huber DL. Absorption, emission and linebreadths: a semihistorical perspective. Rev Mod Phys 1977;49:939-59.

[17] Van Vleck JH, Weisskopf VF. On the shape of collision-broadened lines. Rev Mod Phys 1945;17(2, 3):227-36.

[18] Clough SA, Kneizys FX, Davis RW. Line shape and water vapor continuum. Atmos Res 1989;23:229-41 http:// www.rtweb.aer.com/continuum_frame.html. 
[19] Kuntz M. A new implementation of the humlicek algorithm for the calculation of the voigt profile function. JQSRT 1997;57(6):819-24.

[20] Drayson SR. Rapid computation of the Voigt profile. JQSRT 1976;16:611.

[21] Rosenkranz PW. Absorption of microwaves by atmospheric gases. In: Janssen MA, editor. Atmospheric remote sensing by microwave radiometry. New York: Wiley; 1993. p. 37-90 ftp://mesa.mit.edu/phil/lbl_rt.

[22] Rothman LS, Barbe A, Benner DC, Brown LR, Camy-Peyret C, Carleer MR, et al. The hitran molecular spectroscopic database: edition of 2000 including updates of 2001. JQSRT 2003, 82 (HITRAN special issue).

[23] Gamache RR, Kennedy S, Hawkins R, Rothman LS. Total internal partition sums for molecules in the terrestrial atmosphere. J Mol Struct 2000;517-518:407-25.

[24] Chase MW, Davies CA, Downey JR, Frurip DJ, McDonald RA. Janaf thermochemical tables, 3rd ed. JPCRD 1985;14:1274.

[25] Fischer J, Gamache RR, Goldman A, Rothman LS, Perrin A. Total internal partition sums for molecular species on the 2000 edition of the HITRAN database. JQSRT 2003;82:401-12.

[26] Rosenkranz PW. Interference coefficients for the overlapping oxygen lines in air. JQSRT 1988;39:287-97.

[27] Rosenkranz PW. Water vapor microwave continuum absorption: a comparison of measurements and models. Radio Sci 1998;33(4):919-28 (correction in 34, 1025, 1999), ftp://mesa.mit.edu/phil/lbl_rt.

[28] Cruz Pol SL, Ruf CS, Keihm SJ. Improved 20- to 32-GHz atmospheric absorption model. Radio Sci 1998;33:1319-33 Updated version can be downloaded from http://ece.uprm.edu/ pol/Atmosphere.html.

[29] Liebe HJ. An updated model for millimeter wave propagation in moist air. Radio Sci 1985;20:1069-89.

[30] Liebe HJ, Layton DH. Millimeter-wave properties of the atmosphere: laboratory studies and propagation modelling. Technical Report 87224, US Department of Commerce, National Telecommunications and Information Administration, Institute for Communication Sciences, 1987. 80p.

[31] Liebe HJ. MPM — an atmospheric millimeter-wave propagation model. Int J Inf Millim Waves 1989;10(6):631-50.

[32] Liebe HJ, Rosenkranz PW, Hufford GA. Atmospheric 60-GHz oxygen spectrum: new laboratory measurements and line parameters. JQSRT 1992;48(5/6):629-43.

[33] Liebe HJ, Hufford GA, Cotton MG. Propagation modeling of moist air and suspended water/ice particles at frequencies below $1000 \mathrm{GHz}$. In: AGARD 52nd Specialists Meeting of the Electromagnetic Wave Propagation Panel, Palma de Mallorca, Spain; 1993. ftp://ftp.its.bldrdoc.gov/pub/mpm93/.

[34] Borysow A, Frommhold L. Collision induced rototranslational absorption spectra of $\mathrm{N}_{2}-\mathrm{N}_{2}$ pairs for temperatures from 50 to $300 \mathrm{~K}$. Astrophys J 1986;311:1043-57 http://www.astro.ku.dk/aborysow/programs/.

[35] Ma Q, Tipping RH. Water vapor millimeter wave foreign continuum: a Lanczos calculation in the coordinate representation. J Chem Phys 2002;117:10581-96.

[36] Kuhn T. Atmospheric absorption models for the millimeter waver range. PhD thesis, University of Bremen, 2003.

[37] Kuhn T, Bauer A, Godon M, Buehler SA, Kuenzi K. Water vapor continuum; absorption measurements at $350 \mathrm{GHz}$ and model calculations. JQSRT 2002;74(5):545-62.

[38] Goody RM, Yung YL. Atmospheric radiation, theoretical basis, 2nd ed. Oxford: Oxford University Press; 1989.

[39] Eriksson P, Jimenez C, Murtagh D, Elgered G, Kuhn T, Buehler S. Measurement of tropospheric/stratospheric transmission at $10-35 \mathrm{GHz}$ for $\mathrm{H}_{2} \mathrm{O}$ retrieval in low earth orbiting satellite links. Radio Sci 2003;38(4):8069 doi:10.1029/2002RS002638.

[40] Elgered G. Tropospheric radio-path delay from ground-based microwave radiometry. In: Janssen M, editor. Atmospheric remote sensing by microwave radiometry, 1st ed. New York: Wiley-Interscience; 1993. p. 215-58 [chapter 8].

[41] Kyle T. Atmospheric transmission, emission and scattering. Oxford: Pergamon Press; 1991.

[42] Balluch M, Lary D. Refraction and atmospheric photochemistry. J Geophys Res 1997;102:8845-54.

[43] Rodgers CD. Inverse methods for atmospheric sounding: theory and practise, 1st ed. Singapore: World Scientific Publishing; 2000.

[44] Rodgers CD. Characterization and error analysis of profiles retrieved from remote sounding measurements. J Geophys Res 1990;95:5587-95.

[45] Chance K, Jucks KW, Johnson DG, Traub WA. The smithsonian astrophysical observatory databese sao92. J. Quant. Spectrosc. Radiat. Transfer 1994;52:447-57. 
[46] Jacquinet-Husson N, Arie E, Ballard J, Barbe A, Bjoraker G, Bonnet B, Brown LR, Camy-Peyret C, Champion JP, Chedin A, Chursin A, Clerbaux C, Duxbury G, Flaud J-M, Fourrie N, Fayt A, Graner G, Gamache R, Goldman A, Golovko V, Guelachvili G, Hartmann JM, Hilico J, Hillman J, Lefevre G, Lellouch E, Mikhailenko SN, Naumenko OV, Nemtchinov V, Newnham DA, Nikitin A, Orphal J, Perrin A, Reuter DC, Rinsland CP, Rosenmann L, Rothman LS, Scott NA, Selby J, Sinitsa LN, Sirota JM, Smith AM, Smith KM, Tyuterev VG, Tipping RH, Urban S, Varanasi P, Weber M. The 1997 spectroscopic GEISA databank. J. Quant. Spectrosc. Radiat. Transfer 1999;62:205-54.

[47] Jacquinet-Husson N, Scott NA, Garceran K, Armante R, Chedin A. The 2003 edition of GEISA: A spectroscopic database system for the second generation vertical sounders radiance simulation, in: Proceedings of the 13th International TOVS Study Conference (ITSC-13) 28 October - 4 November.

[48] Eriksson P, Jimenez C, Buehler SA. Qpack, a general tool for instrument simulation and retrival work. J Quant spectrosc Radiat Transfer, doi: 10.1016/j.jqsrt.2004.05.050. 